Gynäkol Rundsch 1991;31(Suppl 2):I-XIV

\title{
Contents, Vol. 31, Supplement 2, 1991
}

Streitmatter, Anna; Jawny, J.; Riedel, K.; Arnold, E.; Wünsch, P. H.: Die primäre zytostatische Behandlung des Vaginalkarzinoms 231

Freie Vorträge: Zervixkarzinom

Stumpfe, M.; Maaßen, V.; Baltzer, J.: Erste Ergebnisse der adjuvanten Chemotherapie des operierten Zervixkarzinoms 235

Lahousen, M.; Haas, J.: Adjuvante Chemotherapie beim radikal operierten Zervix karzinom 237

Batka, M.; Staudach, A.; Haidinger, Maria; Doringer, E.: Magnetresonanz-Staging als Entscheidungshilfe bei der Therapie des Zervixkarzinoms 239

Luschin-Ebengreuth, G.; Girardi,F.; Haas, J.: Zervixkarzinome bei jungen Frauen . . 241

Stumpfe, M.; Dimpfl, Th.; Genz, T.: Der Einfluß histomorphologischer Parameter auf die Entstehung von Lymphozelen nach Radikaloperationen gynäkologischer Malignome 243

Winter, R.: Radikalchirurgie am Scheidenblindsack 245

Schatten, C; Pateisky, N.; Barrada, M.; Enzelsberger, H.; Czerwenka, K.; Kubista, E.: Immun-Lymphszintigraphie in der gynäkologischen Onkologie 247

Enzelsberger, H.; Kurz, Ch.; Schatten, C.; Mittermayer, F.; Vavra, N.: Zum Einfluß der Bestrahlungstherapie auf urodynamische Parameter bei Kollum-Ca-Patientinnen 249

Eisenmenger, M.; Höbarth, K.; Eglau, K.; Hofbauer, H,: 1st die supravesikale Harnableitung beim terminalen gynäkologischen Karzinom indiziert? 251 Freie Vorträge: Korpuskarzinom

Seelbach-Göbel, Birgit; Rempen, A.; Kristen, P.: Vaginalsonographie am Endometriuminder Postmenopause. Erste Ergebnisse einer prospektiven Studie 253 Schweighart, Cornelia; Schramm, Th.; Kürzl, R.; Stuckert-Klein, A. C: Endometrium-Karzinom: Diagnostische Validität eines vaginalsonographischen Screen ings 256

Vavra, N.; Sevelda, P.; Schemper, M.; Kucera, H.; Weghaupt, K.: Die Bedeutung von Prognosefaktoren bei lymphonodektomierten Patientinnen mit Endometriumkarzinom 258

Dörffler, P.; Caffier, H: 1st bei einer adenomatösen Hyperplasie des Endometriums eine Kontrollabrasio indiziert? 261

Girardi, F., Petru, E., Haas, J.: Der Lymphknotenstatus bei Korpus- und Zervixkarzi nom 263

Schünemann, H; Strigl, P.; Hugo, R. von: Korpuskarzinom in der Nachsorge 266 Feiks, A.; Grünberger, W.: Therapie der atrophen Kolpitis - 1st eine Reduktion der Östrogendosis bei lokaler Anwendung möglich? 268

Freie Vorträge: Ovarialkarzinom 
Tamussino, K.; Petru, E.: Prätherapeutische Skalenusbiopsie beim Ovarialkarzinom. . 272 Petru, E.; Pickel, H; Lahousen, M.; Heydarfadai, M.; Auner, J.: Paraaortale Lymphknoten-Rezidive bei Stromatummoren und Keimzelltumoren des Ovars 274

Inhalt

Editorial XIII

Graeff, H.; Burghardt, E.: Eröffnung der Tagung 1

Burghardt, E.: Begrüßung 3

1. Hauptthema: Begleitende Maßnahmen bei onkologische $\pi$ Patientinnen

Referate

Onkologische Heilbehandlung und Rehabilitation 5

Schröck, R.; Stepien, J.; Schwiersch, M.: Onkologische Heilbehandlung und Rehabili tation 5

Staufer, F.: Onkologische Nachsorge in der gynäkologischen Praxis, Bayerisches

Nachsorgemodell 11

Thieme, Ch.: Ergebnisse der onkologischen Dokumentation des Bayerischen Nachsorgemodells 17

Sevelda, P.; Vavra, N.; Barrada, M.; Seifert, M; Wagner, T.; Salzer, H.: Supportive

Maßnahmen zur zytostatischen Therapie gynäkologischer Malignome 21

Jänicke, F.; Kuhn, W.; Hölscher, M.; Graeff, H.: Sekundäre operative Interventionen beim Ovarialkarzinom 26

2. Hauptthema: Urologische Früh- und Spätkomplikationen gynäkologischonkologischer Eingriffe

Referate

Kölbl, H.: Die Bedeutung der praeoperativen Diagnostik für die Praevention postoperativer Komplikationen an den ableitenden Harnwegen 35

Ralph, G.; Tamussino, K.; Michelitsch, L.: Einfluß der operativen Radikalität auf die Entstehung postoperativer urologischer Komplikationen 41

Schüßler, B.: Therapie funktioneller Störungen des unteren Harntraktes nach Radikalchirurgie im kleinen Becken 46

Pateisky, N.; Vavra, N.: Isotopennephrographie in der Verlaufskontrolle von Zervixkarzinompatientinnen nach Radikaloperation 52

3. Hauptthema: Androgenisierung

Referate

Urdl, W.: Androgenisierung. Eine Übersicht 55

Breckwoldt, M.; Wieacker, P.: Androgenisierung und Androgeninsensitivität $\quad 60$ Inhalt

VI

Huber, J. C: Somatomedin und Somatostatin beim polyzystischen Ovar $\quad 66$ Vytiska-Binstorfer, Elisabeth: Zur Inzidenz der Hyperandrogenämie 69

Fischl, F.; Müller-Tyl, E.; Bieglmayer, Ch.; Reinthaller, A.: Hormonelle Stimulation hyperandrogenämischer Patientinnen bei IVF 74

4. Hauptthema: Vorhersagbarkeit fetaler Gefährdung und prädikativer Wert perinatologischer Untersuchungsmethoden

Referate

Kürzl, R.: Vorhersagbarkeit fetaler Gefährdung und prädiktiver Wert perinatologischer Untersuchungsergebnisse: Eine Herausforderung des Geburtshelfers .... 79 
Schneider, K. T. M.: Antenatale Untersuchungsmethoden zur Erkennung fetaler Gefährdung 87

Lassmann, R.; Steiner, H.; Schaffer, H.; Staudach, A.: Wertigkeit präpathologischer und pathologischer Dopplerbefunde im Vergleich zu CTG, Hormon- und Asphyxieparametern 92

Steiner, H.; Schaffer, H.; Lassmann, R.; Batka, M,; Staudach, A.: Validität von

Dopplerbefunden in Hinblick auf das Fetale Outcome 99

Freie Vorträge: Geburtshilfe

Gyr, Th.; Berclaz, G.; Markus, D.; Bratschi, H. U.; Schneider, H,: Prophylaktische

Tokolyse beim frühen vorzeitigen Blasensprung 105

Berclaz, G.; Gyr, Th.; Bratschi, H. U.; Markus, D.; Schneider, H.: Lungenreifungsin-

duktion beim frühen vorzeitigen Blasensprung 108

Kästner, Beate; Schenk, H.; Weise, W.: Die diagnostische Zuverlässigkeit des Neura-

minsäure/Protein-Quotienten im Fruchtwasser als Indikator für die fetale Reife. . 111 Rammer,

Edith; Friedrich, F.; Simml, Maria: Liegekuren zur Prophylaxe bei drohen-

den Frühgeburten 113

Lechner, W.; Wartusch, B.; Allerberger, F.; Guggenbichler, G.; Hagen, G.; Solder,

E.; Kölle, D.: Einfluß von Streptokokken auf die uterine Aktivität 115

Gmoser, G.; Girardi, F.; Mayer, H. O.; Hermann, J.; Haas, J.: Das Stützpessar-eine

Therapiemöglichkeit bei vorzeitiger Eröffnung des Verschlußapparates 117

Riehn, A.; Szendzielorz, D.; Riehn, Frigga; Kurt, Edeltraud; Justus, Barbel: Geburts-

hilfliche Parameter und Morbidität sehr kleiner Neugeborener $\kappa 11500$ g 119

Buschmann, J.; Knitza, R.: Oxycardiotokografie (OCTG). Ein neues Verfahren zur

Messung der fetalen Sauerstoffsättigung sub partu. Einführung in die Methode . . 122 Knitza, R.;

Buschmann, J.: Oxycardiotokografie (OCTG). Ein neues Verfahren zur

Messung der Fetalen Sauerstoffsättigung sub partu. Anwendung der Methode. . . 125

Rudelstorfer, R.: Neue und etablierte Methoden zur Geburtsüberwachung im Ver

gleich ihrer Vorhersagekraft fetaler Gefahrenzustände

127

Adelwöhrer, N. E.; Gmoser, G.; Rollett, H.; Dimai, H. P.; Seibert, F.; Winter, R.:

Unterschiedliche Dosierungsintervalle bei der oxytocinunterstützten Wehentätig-

keit - eine retrospektive Studie

129

Stumberger, E.; Burkl, J.; Wiborny, R.: Optimierung des Geburtszeitpunktes mit

Prostin E 2132

Inhalt

VII

Knitza, R.; Eberl-Lehmann, P.; Artmeier, U.; Finsterer, U.: Patientengesteuerte lumbale Katheterperiduralanästhesie versus kontinuierliche. Perfusorkatheterperiduralanästhesie - Erste Ergebnisse 134

Gloning, K.-Ph.; Schramm, Th.; Ostermayer, Eva; Brusis, E.; Kuß, E.: Schwere Wachstumsretardierung: Ergebnisse der Untersuchung fetaler Blutproben (Cordozentese) 136

Schneider, K. T. M.; Amberg-Wendland, D.; Renz, S.; Fürstenau, U.: Prospektiv randomisierte Untersuchung zum klinischen Wert der Dopplersonographie als Screeningverfahren 139

Voigt, H. J.; Meyer, M.: Diskriminanz-Analyse dopplersonographischer Daten zur geburtshilflichen Risikoeinschätzung 141 
Mauch, E. D.; Voigt, H. J.; Beinder, E.: Doppler-Sonographie versus herkömmliche Überwachungsmethoden - Vergleich der Testvalidität 144

Beinder, E.; Voigt, H. J.; Mauch, E.: Klinische Wertigkeit der Dopplersonographie . . 147

Schramm, Th.; Pflaumer, A.; Salanki, Ildiko; Gloning, K.-Ph.: Differentialdiagnose des biometrisch kleinen Kindes mittels Dopplersonographie 150

Tulzer, G.; Gudmundsson, S.; Huhta, J. C; Wood, D. C,; Tews, G,: Wert des

Dopplers in der Evaluierung und Prognose von Feten mit nicht-immunologi-

schem Hydrops fetalis 152

Kubista, E.; Enzelsberger, H.; Skodler, W. D.: Der Einfluß des TNS auf sonographische Funktionsparameter bei Plazentainsuffizienz 154

Krone, S.; Wisser, J,: Vaginalsonographische Abklärung der Placenta praevia 155

Rempen, A.: Pränatale Diagnostik und Dopplersonographische Uberwachung einer

Zwillingsschwangerschaft mit Acardius

Ostermayer, Eva; Schweighardt, Cornelia; Schramm, Th.; Gloning, K.-Ph.; Steldin-

ger, R.: Syndrom der caudalen Regression bei dem Fetus einer Typ I Diabetike-

rin: Fallbericht 159

Hönigl, W.; Zach, M. S.; Rosenkranz, W.; Mayer, H. O.; Häusler, M. C. H.;

Walcher, W.; Oberwaldner, B.; Weiß, P. A. M.: Schwangerschaft bei Mucovisci-

dose 162

Walcher, W.; Pusch, H. H.; Hönigl, W.; Mayer, H. O.; Häusler, M.; Ralph, G.:

Unfall in der Schwangerschaft 164

Beck, A.;Vutuc, Ch.;Friedl, H. P.: Müttersterbefälle in Österreich 1975-1989 166

Quittan, Gabriele; Medl, M.; Leodolter, S.: Analyse dopplersonographischer Befunde bei diabetischen Schwangerschaften 169

Schröcksnadel, H.; Sitte, B.; Alge, A.; Waitz-Penz, A.; Abfalter, E.: Prädiktiver Wert der Hämolyse für den fetomaternalen Outcome bei Patientinnen mit schwangerschaftsinduzierter Hypertonie 174

Schneider, K. T. M.; Dumler, E. A.; Oettle, W.; Schöffel, J.; Graeff, H.: Vier Jahre

Erfahrung mit der individuellen Qualitätssicherung in der Geburtshilfe 176

Fabrizii, V.; Nowotny, Ch.; Dadak, Ch.; Swoboda, K.; Endler, N.; Derfler, K.;

Balcke, P.: Tubuläre Alterationen bei normaler Gravidität und EPH-Gestose;

Diagnose durch NAG 178

Getz, B.; Heidegger, H.; Hümpfner, A.: Hypertonie in der Schwangerschaft: Möglichkeiten der Differenzierung der verschiedenen Hochdruckformen anhand von

Serum-und Urinparametern 180

Inhalt

VIII

Schröcksnadel, H.; Sitte, B.; Alge, A.; Holböck, E.; Brock, P.: Prognostische

Bedeutung der peripartalen Thrombopenie 181

Ruckhäberle, K.-E.; Viehweg, Brigitte; Fuller, Kerstin; Robel, R.; Faber, R.; For-

berg, J.: Prädiktiver Wert ausgewählter Untersuchungsparameter bei anamnesti-

schem Risiko für Frühgeburt 183

Alge, A.; Sitte, B.; Schröcksnadel, H.; Schwegel, P.: Erfahrungen mit dem Roll-over-

Test im Gestosescreening 186

Menton, M.; Wiest, E.: Erste Erfahrungen mit einer neuen sondengesteuerten CVS-

Technik $\quad 188$ 
Ostermayer, Eva; Gloning, K.-Ph.; Brusis, E.: Amniozentese in der 12.-14. Schwangerschaftswoche: Erste Erfahrungen mit 107 Eingriffen 190

Klein, M.; Bartosch, B.; Beck, A.; Rosen, A.; Schnedl, W.: Die Amniozentese im ersten Schwangerschaftsdrittel 192

Brusis, E.; Gloning, K. Ph.: Erfahrung mit 7127 Frühamniozentesen bei 6924 Schwangerschaften an der I. UFK München in den Jahren 1976-1989 195

Kolben, M.; Kupf, K.; Saks, M.; Fischbach, F,: Bedeutung zervikaler Chlamydieninfektionen in der Schwangerschaft für Mutter und Kind 198

Schieß1, B.; Lack, N.; Schneider, K. T. M.: Der intrauterine Fruchttod - wo versagt die Schwangerenvorsorge? 201

Wierrani, F.; Marcovich, Marina; Pechter, Brigitte; Grünberger, W.: Vorhersehbarkeit fetaler Gefährdung durch ultrasonographisches Screening 203

Fitz, R., Adler, A.: Zuckerbelastung - nur Personalbelastung? Der orale Glukosetoleranztest im Wochenbett 205

Wierrani, F.; Kozak, W.; Schramm, W.; Grünberger, W.: 1st die Transferierungsrate Neugeborener ein Qualitätskriterium perionatologischer Betreuung? 208 Radivojevic, K; Schaller, A.: Isolierte Hautmanifestation eines Amnionbandsyndroms und deren klinische Bedeutung 210

Heidegger, H.; Welponer, F.; Hugo, R. v.; Delucca, A.: Vergleich der Perinatalerhebungen aus dem Jahre 1989 zwischen Bayern und Südtirol 213

Freie Vorträge: Dysplasien von Vulva und Zervix Wartusch, Barbara; Heim, K; Holböck, E.; Weiss, D.; Pinzger, G.: HPV-Inzidenz und -Typenverteilung bei verschiedenen gynäkologischen Patientenkollektiven . 215 Kainz, Ch.; Gitsch, G.; Reinthaller, A.; Kopp, W,; Tatra, G.; Breitenecker, G.: Zervixdysplasien und Human-Papilloma-Virus Infektion bei Prostituierten 217 Heim, K.; Höpfl, R.; Sandbichler, M.; Sepp, N.; Wartusch, B.; Müller-Holzner, E.; Jochmus-Kudielka, I.; Ter Meulen, J.; Gissmann, L.; Fritsch, P.; Dapunt, O.: Intrakutantest zur Erfassung zellulärer Immunreaktionen gegen HPV-16-Proteine bei Patientinnen mit CIN 220

Kainz, Ch.; Gitsch, G.; Reinthaller, A.; Tatra, G.; Breitenecker, G.: Milde Zervixdys plasien: Hat die diagnostische Biopsie einen ,therapeutischen” Effekt? 223 Pinzger, G,; Heim, K.; Holböck, E.; Müller-Holzner, E.; Wartusch, B.; Dapunt, O.: Diagnostik und Therapie der Vulvadystrophie 225

Feiks, A.; Wierrani, F.; Grünberger, W,: Der routinemäßige Einsatz des C02-Lasers an einer großen gynäkologischen Abteilung 230

Inhalt

$\mathrm{X}$

Kuhn, W.; Jänicke, F.; Hölscher, M.; Schüle, G.; Pache, L.; Graeff, H.: Operative Behandlung des Ovarialkarzinoms: Vergleich primärer und sekundärer tumorreduktiver Eingriffe 276

Beck, E.; Schuster, M.; Berg, A.; Jäger, W.; Wildt, L.; Lang, N.: Ototoxizität unter cisplatin-haltiger Chemotherapie bei Patientinnen mit Ovarialkarzinomen 280 Schelling, M.; Jänicke, F.; Senekowitsch, R.; Lörken, A.: Der Tumormarker CA 72-4 beim Ovarialkarzinom im Vergleich mit CA 125 und D-Dimer 286 
Ruppitsch, U.; Petru, E.; Woltsche, M.; Pickel, FL; Lahousen, M.: Bedeutung der prophylaktischen Ovarektomie anläßlich einer Hysterektomie zur Vermeidung eines Ovarialkarzinoms 289

Täubert, D.; Neises, M.; Wischnik, A.; Holm, E,: Supportive enterale Ernährungstherapie bei Zytostatikabehandlung trotz verminderter Resorptionsleistung des

Dünndarms 291

Freie Yorträge: Mammakarzinom

Schweiberer, Sabine; Hegerfeld, R.; Scheidel, P.; Schüßler, B.: Vergleichende Untersuchung zur Optimierung der Drainage nach axillärer Lymphadenektomie 294 Jäger, W.; Merkle, E.; Tulusan, A. H.; Lang, N.: Modifizierte MammakarzinomNachsorge 296 Harbeck, Nadia; Moniwa, N.; Busch, E.; Schmitt, M.; Jänicke, F.; Fellbaum, C; Höfler, H.; Graeff, H.: Durchflußzytometrische DNA-Analyse von reinen Zellkernen aus formalin-fixierten Paraffinschnitten beim primären Mammakarzinom: Korrelation mit anderen Prognosefaktoren 299

Kristen, P.; Kaesemann, H.; Rempen, A.; Beier, H.-J.; Caffier, PL: DNA-ImageZytometrie beim Mamma-Karzinom: Vergleich zu anderen Prognosekriterien . . 302 Schaudig, Katrin; Thomssen, C.; Jänicke, F.; Graeff, FL: Mitoxantron Monotherapie beim metastasierten Mammakarzinom - erste Ergebnisse einer prospektiven randomisierten Studie bei „low risk”-Patientinnen 304 Bergant, A.; Schröcksnadel, H.; Heim, K.; Sander, T.; Abfalter, E.: Die Wertigkeit der Knochenszintigraphie in der Mammakarzinomnachsorge $\quad 307$ Seifert, M.; Adler, A.; Obermair, A.; Hartmann, B.; Denison, U.; Kubista, E.; Sevelda, P.: Möglichkeiten und Ziele in der Tumornachsorge beim Mammakarzi nom 309

Freie Vorträge: Gynäkologische Urologie

Hesse, U.; Debus-Thiede, G.; Meier, B.; Schüßler, B.: Kernspintomographie des Beckenbodens - eine Erweiterung unserer anatomischen Vorstellung 312 Voigt, R.; Sommer, H.; Halaska, M.; Stech, D.; Nöschel, H.: Urodynamische Untersuchungen nach Operation, Strahlenbehandlung und Zytostase gynäkologischer Malignome $\quad 313$

Luschin-Ebengreuth, G.; Ralph, G.; Tamussino, K.: Die endoskopische Blasenhalsanhebung-Klinische, urodynamische und radiologische Ergebnisse 315 Tamussino, K.; Ralph, G.: Operative Therapie der Streßinkontinenz nach radikaler Hysterektomie 317

Inhalt

XI

Methfessel, H. D.; Wolters, M.; Lampe, D.; Methfessel, G.; Ketscher, K.: Ureterläsionen bei der radialen Hysterektomie mit pelviner Lymphonodektomie 319 Spitzer, D.; Staudach, A.; Joos, H.; Steiner, H.: Urologische Komplikationen bei gynäkologischen Tumoreingriffen 320

Dimpfl, Th.; Stumpfe, M.; Genz, T.: Abdominale gynäkologische Malignomoperationen: Folgen und Komplikationen 323

Kurz, Christine; Enzelsberger, H.; Adler, A.; Knogler, W.: Intravesikal appliziertes Östriol als mögliche Therapieform der sensorischen Urgeinkontinenz 326 Steck, T.; Petraki, S.; Baseler, C; Albert, P. J.; Wernze, H.: 1st die gesteigerte 
Freisetzung von LH nach Gabe von LHRH ein zuverlässiger Indikator für das Syndrom der polyzystischen Ovarien? 328

Rossmanith, W. G.; Schramm, S.; Benz, R.; Lauritzen, C.: Wertigkeit des GnRHDoppelstimulationstestes bei der Differenzierung der Oligo-Amenorrhoe 331 Rossmanith, W. G.; Keckstein, J.; Spatzier, K.; Lauritzen, C: Endokrine Effekte der Laservaporisation der Ovarien bei Frauen mit polyzystischen Ovar-Syndrom . . 333 Strowitzki, T.; Korell, M.; Hepp, H.: Sterilitätstherapie bei PCO-Patientinnen 335 Lorenz-Eberhardt, G.; Strampfer, H.; Urdl, W.; Hönigl, W.: Spektrophotometrische Analysen von Follikelflüssigkeiten im IVF Programm 338

Freie Vorträge: Pelviskopie

Fiedler, K.; Hirsch, P.; Krüsmann, G.; Würfel, W.: Stellenwert der pelviskopischen Tuboskopie in der Fertilitätsmikrochirurgie 341

Freude, G.; Leodolter, S.; Wang-Artner, B.: Erste Erfahrungen mit der hysteroskopischen Kathetertechnik zur Rekanalisierung proximaler Tubenverschlüsse 343 Wiborny, R.: Die Therapie der Tubargravidität durch hysteroskopische Gabe von Prostaglandin 345

Deckardt, R.; Jänicke, F.; Kuhn, W.: Therapie der Tubargravidität mit Prosta glandin 246

Fiedler, K.; Krüsmann, G.; Würfel, W.: Die operative Pelviskopie in der Behandlung der Extrauteringravidität nach in vitro Fertilisation und Spontankonzeption .... 348 Siebzehnrübl, E.; Paterok, E.; Todorow, S.; Wildt, L.; Lang, N.: Die Behandlung der Tubargravidität per laparoskopiam: PGF2 $\alpha$ Tnjektion contra chirurgische Inter vention 351

Rudelstorfer, B.: Wundverschluß mit Fibrinklebung in der pelviskopischen Chirurgie. . 353

Lahodny, J.: Ethi-Endo-Clip-Naht - Ein Fortschritt in der pelviskopischen Chirurgie. . 355

Freie Vorträge: Psychosomatik

Greimel, Elfriede; Egger, J.; Freidl, W.; Hofwarter, B.; Lahousen, M.: Krankheitsverarbeitung von Frauen mit Genitalkarzinomen 358

Winkler, Lieselotte; Graeff, H.: Hyperemesis als Abgrenzungsversuch - ein Fallbericht 360

Fischer, Ivo: Zur Frage psychischer Einflüsse auf die Entscheidung des Brustkarzinoms 362

Walcher, W.; Wisiak, U. V,; Glawischnig, M.: Wer braucht Geburtsvorbereitung? . . 264

Brandt, K.; Zech, H.: Psychologische Kurzzeitpsychotherapie bei Vaginismus 366 Inhalt

XII

Freie Vorträge: Grundlagenorientierte klinische Forschung

Kurbacher, C. M.; Breuel, C; Kurbacher, J.; Jäger, W.; Wildt, L.; Lang, N.:

Prognostische Bedeutung des Tumorzellnachweises in Exsudaten und Peritonealspülungen durch Monolayer-Kulturen 369

Gitsch, G.; Kohlberger, Petra; Kölbl, H.; Krepler, R.; Breitenecker, G.: Immunhistochemische Differenzierung von Granulosazelltumoren und Ovarialkarzinomen . 370 Marth, C.; Lang, T.; Daxenbichler, G.: Beeinflussung des EGF-Rezeptors durch eine zytostatische Behandlung 373

Bieglmayer, Ch.; Hofer, G.; Reinthaller, A.; Kainz, Ch.; Janisch, H.: Einfluß

hormoneller Kontrazeptiva auf den Eicosanoidgehalt des Menstrualblutes 375 
Czerwenka, K. F.; Zeillinger, R.; Kury, F.; Manavi, M.; Speiser, P.; Peschke, M.; Steinhart, U.; Hosmann, J. W.; Kubista, E.: Anwendung der PolymeraseKettenreaktion (PCR) als Zusatzdiagnostik in der Gynäko-Onkologie $\quad 378$ Heim, K.; Heis, W.; Dapunt, O.: Evaluierung eines integrierten geburtshilflichen Datenverarbeitungsprogramms auf Personalcomputer $\quad 380$

Kentner, P.: Fetales und maternales Risiko bei 782 Geburten nach vorausgegangener Schnittentbindung $\quad 384$

Klieber, R.; Pastner, E.; Schwegel, P.; Kirchler, H; Alge, A.; Sitte, B.; Schröcksnadel, H.: Auswirkungen schwerer EPH-Gestosen auf das neonatale Blutbild .... 386

Kosian, K.: Therapie vaginaler Infektionen bei postmenopausalen Patientinnen 388

Manavi, M.; Czerwenka, K. F,; Schurz, B.; Knogler, W.; Kubista, E.; Reinold, E.:

Latente zervikale Virusinfektion als mögliche Ursache des Frühaborts 389

Mitterschiffthaler, G.; Schlager, A.; Schröcksnadel, H.: Optimiert die lumbale Epidu ralanalgesie die Hämodynamik und Nierendysfunktion bei EPH-Gestose? 391

Mitterschiffthaler, G.; Schlager, A.; Schröcksnadel, H.: Lumbale Epiduralanalgesie bei EPH-Gestose: fetales und maternelles Risiko 394

Seifert, M.; Wierrani, F.; Kubista, E., Spona, J.: Vergleich von Östrogen- und Progesteronrezeptorengehalt von primären Mammakarzinomen ohne skelettaler oder viszeraler Metastasierung mit der Serumkonzentration der Tumormarker MCA, CEA und CA 15-3 zumZeitpunkt der Operation 397

Wiborny, R.: Die hysteroskopische Ballonkatheterplastik 398

Wirth, U.; Rossmanith, W. G.; Sasse, V.; Lauritzen, C: Unterschiedhche Wirkungen von GnRH-Analogen auf die Androgenkonzentrationen bei Frauen 400

Würfel, W.; Beckmann, M. W.; Austin, R.; Steck, T.; Herzog, Ute; Link, Ulla; Albert, P. J.: In vitro Untersuchungen zur Regulation der Sekretion und Synthese von humanem Choriongonadotropin (hCG) durch Prolaktin (PRL) in der humanen Terminplazenta 402

Autorenregister

406

Editorial

Es ist eine bewährte und liebgewordene Tradition, daß die Bayeri-sche und Österreichische Gesellschaft jedes zweite Jahr gemeinsam tagen. Es ist dies anregend, stimulierend und fruchtbringend zugleich.

In diesem Jahr waren die Österreicher Gast in München. Der President der Bayerischen Gesellschaft, Professor H. Graeff und sein Tagungs-sekretär, OA R. Deckard haben, gemeinsam mit dem Präsidenten der Österreichischen Gesellschaft, Professor E. Burghardt das Programm gestaltet.

Die Hauptthemen waren hochaktuell: „Begleitende Maßnahmen bei onkologischen Patientinnen”, „Urologische Komplikationen gynäkolo-gisch-onkologischer Eingriffe”, „Androgenisierung” und schließlich „Vorhersagbarkeit fetaler Gefährdungen und prädiktiver Wert perinato-logischer Untersuchungsmethoden". Zu jedem dieser Themen wurden jeweils sich gegenseitig ergänzende Referate gehalten, sodaß jedes voll-ständig und kompetent abgedeckt wurde.

Besonders groß war auch diesmal die Zahl der „Freien Vorträge”, die in die Bereiche „Geburtshilfe”, „Dysplasien von Vulva und Zervix”, „Zervixkarzinom”, „Korpuskarzinom”, „Ovarialkarzinom”, „Mamma-karzinom”, „Psychosomatik”, „Endokrinologie”, „Gynäkologische 
Uro-logie”, „Pelviskopie” und „Grundlagenorientierte klinische Forschung” gegliedert war; dazu kamen noch zahlreiche Poster. Auch alle diese Beiträge sind in komprimierter Form in diesem Kongreß-Supplement zusammengefaßt, sodaß der Band auf den stattlichen Umfang von über 400 Seiten angewachsen ist.

Dieser umfangreiche Band konnte auch in diesem Jahr nur mit der Hilfe unserer Sponsoren herausgebracht werden, ihnen gilt unser ganz besonderer Dank. Wir wissen es sehr zu schätzen, daß diese Firmen ihre Verbundenheit mit unserem Fachgebiet auch auf diese Weise dokumentieren wollten und unserer Einladung zur Sponsorenschaft gefolgt sind.

Unser Dank gilt in diesem Zusammenhang aber auch den MitarbeiInhalt

XIV

tern der Druckerei Berger \& Söhne in Horn, Niederösterreich, und nicht zuletzt auch dem Verlag S. Karger in Basel, denn nur mit dieser Hilfe war es möglich, den Band in so kurzer Zeit nach der Tagung bereits zur Auslieferung zu bringen; eine Forderung, die wegen der Aktualität des Gebotenen unbedingt als notwendig erachtet wird.

E. Reinold 